\title{
La fecundidad en las áreas rurales y urbanas de México
}

\author{
Irma Olaya García y Garma*
}

Los objetivos de este trabajo son hacer un estudio comparativo para observar la evolución de la fecundidad en las áreas urbanas y rurales del país y al mismo tiempo analizar si la anticoncepción en las zonas rurales podría practicarse significativamente aun con un desarrollo económico incipiente.

Para cumplir con el objetivo propuesto se utilizan los datos de seis encuestas realizadas entre 1969 y 1987 y se calculan, para las áreas rurales y urbanas del país, las tasas específicas de fecundidad marital y el indice $m$ de control de fecundidad desarrollado por Coale y Trussel. Eí estudio nos permite ver lo siguiente: a) que durante el periodo de observación, se perdieron las condiciones de fecundidad natural que existían en 1969 en las áreas rurales del país y que, a través del tiempo, los descensos en la fecundidad y consecuentemente los ascensos en el uso de anticonceptivos se van incrementando; b) que en las áreas urbanas la anticoncepción es ampliamente practicada y su uso se extiende cada vez más, y c) al observar el comportamiento del descenso registrado en las zonas rurales en el periodo 1976-1982, la teoría de la modernización pierde fuerza ya que, al menos en algunas ocasiones, el ritmo de aumento en la limitación del tamaño de la familia ha sido mayor en las áreas rurales que en las urbanas. Aun cuando nuestro postulado hasta cierto punto se confirma, es probable que un descenso sostenido de la fecundidad en las zonas más deprimidas del país sólo pueda lograrse con cambios socioeconómicos sustantivos.

\section{Introducción}

En un estudio basado en los datos de la encuesta PECFAL-Rural (1969-1970) que forma parte del Programa de Encuestas Comparativas de Fecundidad en América Latina, se observaron altos niveles de fecundidad que se aproximaban a lo que los demógrafos llamamos condiciones de "fecundidad natural", es decir que en la mayoría de las parejas no existía un intento consciente de regular el número y espaciamiento entre sus hijos.

En el mismo estudio se infirió que gran parte de la población que habitaba en esas localidades se encontraba sumergida en un ambiente cultural que podría ser calificado de premoderno y tradicional. El nivel educativo era bastante bajo y, en general, la mu-

* Profesora-investigadora del Centro de Estudios Demográficos y de Desarrolo Urba no de El Colegio de México. 
jer campesina mantenía solamente un contacto marginal con el mundo externo que la rodeaba. En consecuencia, estaba lejos de percibir que existían otras formas de vida.

Se observó que la alta fecundidad era concurrente con ciertas características entre las cuales destacan las siguientes: las mujeres contraían matrimonio a temprana edad, experimentaban cortos intervalos entre nacimientos, aparentemente eran partidarias de las familias grandes y la mayoría de ellas pensaban que las condiciones económicas por las que pasaban no mejorarían teniendo menos hijos de los que tenían. Igualmente consideraban como "buenas" todas aquellas condiciones que conducen a una alta fecundidad: casarse jóvenes, tener hijos a intervalos cortos, y largos periodos de reproducción.

Por otro lado, aun cuando $61 \%$ de las mujeres casadas o unidas con uno o más hijos sobrevivientes declaró que no quería tener más hijos, sólo un 10\% había hecho uso de la anticoncepción. Un gran número de ellas desconocía la posibilidad de planear su familia y muchas otras padecían conflictos personales - principalmente religiosos y de salud - para llevarla a cabo. En consecuencia, la aceptación hacia el control de la natalidad era escasa, el rechazo hacia el aborto muy enérgico y el uso de los anticonceptivos limitado. Respecto a este último punto se observó una relación positiva entre la educación, el tamaño de localidad de residencia, el nivel socioeconómico y las prácticas para limitar el tamaño de la familia (García, 1976; García y Garma, 1980).

Los resultados de esta encuesta (1969-1970) nos condujeron a plantear la siguiente hipótesis: si el uso de anticonceptivos en las áreas rurales continuaba asociado a las mejores condiciones socioeconómicas, como apareció en el estudio, su propagación sería lenta y el descenso de la fecundidad no ocurriría pronto. Sin embargo, debido a que la motivación para controlar la fecundidad - manifestada en el deseo de no tener más hijos- ya existía en la mayoría de las mujeres, la planificación familiar podría ganar fuerza independientemente de las condiciones socioeconómicas y efectuarse el descenso en el tamaño de la familia aún sin que ocurriera un mejoramiento socioeconómico radical. El objetivo del presente estudio es comprobar la validez de esta hipótesis mediante un análisis del cambio sufrido en la fecundidad en el periodo 1969-1987 en las áreas rurales y urbanas del país. ${ }^{1}$

\footnotetext{
${ }^{1}$ En este trabajo, salvo en casos específicos en los cuales aparece la aclaración, el límite adoptado para diferenciar las localidades rurales de las urbanas es el de 20000 habitantes.
} 


\section{Fuentes de datos}

Además de los censos y de las estadísticas vitales, los demógrafos utilizamos la información recolectada en diversas encuestas, es decir, los resultados de entrevistas practicadas a un grupo o muestra representativa de toda la población. Este hecho nos permite disponer de un valioso caudal de datos que se potencializa por el advenimiento de los procesos electrónicos, de nuevos recursos estadísticos y del desarrollo de nuevas técnicas de análisis. Los estudiosos de la población nos encontramos en una situación privilegiada que nos permite conocer no sólo los niveles, tendencias y diferenciales de las variables demográficas, sino también profundizar en su estudio incorporando en el análisis variables sociales y económicas.

Para cumplir con el objetivo propuesto antes contamos con la información proveniente de seis encuestas realizadas entre 1969 y 1987 (cuatro de ellas a nivel nacional y dos enfocadas sólo a las comunidades rurales). Las fechas cuando fue llevado a cabo el levantamiento, así como el número de casos en cada una de las encuestas que se utilizan en este estudio se descri en en el cuadro 1. En virtud de que todas ellas han pasado una o varias pruebas de evaluación, los datos que se manejan en el presente trabajo han sido tomados, sin modificación alguna, de las fuentes señaladas en cada caso.

El cálculo de las tasas específicas de fecundidad marital ${ }^{2}$ se efectuó utilizando la información sobre la historia reproductiva e historia de matrimonios o uniones proporcionada por las entrevistas en las diferentes encuestas. Se tomó en consideración la fecha del matrimonio y la del nacimiento de cada uno de los hijos vivos, para las mujeres casadas o unidas que reportaron una sola unión al momento de la encuesta. De igual forma calculamos el porcentaje que representa cada una de las tasas cuando la correspondiente al grupo 20-24 se hace igual a 100. En general se manejan datos que constituyen el promedio de los tres años previos a la fecha de cada encuesta.

\footnotetext{
${ }^{2}$ Tasas especificas de fecundidad marital $\left(x+{ }_{4} f x\right)$ se definen como el promedio de nacimientos ocurridos a las mujeres casadas o unidas en el grupo de edad correspondiente.
} 
CUADRO 1

Descripción de las encuestas que se han realizado en México entre 1969 y 1987

\begin{tabular}{|c|c|c|}
\hline Encuesta & Trabajo de campo & $\begin{array}{c}\text { Mujeres } \\
\text { entrevistadas }\end{array}$ \\
\hline \multicolumn{3}{|l|}{ 1. Encuesta de Fecundidad } \\
\hline $\begin{array}{l}\text { Rural en México, } 1969 \\
\text { (Localidades con menos de } \\
20000 \text { habitantes) }\end{array}$ & $\begin{array}{l}\text { Diciembre de } 1969 \\
\text { a marzo de } 1970\end{array}$ & 3000 \\
\hline 2. Encuesta Mexicana de & Junio de 1976 & \\
\hline $\begin{array}{l}\text { Fecundidad } 1976 \text { (DCE, } \\
\text { 1979) }\end{array}$ & a marzo de 1977 & 7310 \\
\hline \multicolumn{3}{|l|}{ 3. Encuesta Nacional de } \\
\hline $\begin{array}{l}\text { Mótodos Anticonceptivos, } \\
1978 \text { (CPNPF, 1979; no se } \\
\text { incluyó en el estudio) }\end{array}$ & $\begin{array}{l}\text { Julio a octubre } \\
\text { de } 1978\end{array}$ & 4492 \\
\hline \multicolumn{3}{|l|}{ 4. Encuesta Nacional de } \\
\hline $\begin{array}{l}\text { Prevalencia en el Uso de } \\
\text { Métodos Anticonceptivos }\end{array}$ & Septiembre a & \\
\hline $\begin{array}{l}\text { con Módulo Fecundidad/ } \\
\text { Mortalidad, } 1979 \text { (CPNPF, } \\
\text { 1980) }\end{array}$ & diciembre de 1979 & 20482 \\
\hline \multicolumn{3}{|l|}{ 5. Encuesta Rural de } \\
\hline $\begin{array}{l}1981 \text { (localidades menores } \\
\text { de } 2500 \text { habitantes según } \\
\text { el Censo de 1970) (JSPF, } \\
\text { 1982) }\end{array}$ & $\begin{array}{c}\text { Septiembre a } \\
\text { octubre de } 1981\end{array}$ & 8103 \\
\hline 6. Encuesta Nacional & Febrero y marzo & \\
\hline $\begin{array}{l}\text { Demográfica, } 1982 \\
\text { (Conapo, 1983) }\end{array}$ & 097 & 10205 \\
\hline 7. Encuesta Nacional sobre & 23 de febrero a 31 & \\
\hline Fecundidad y Salud (1987) & de mayo de 1987 & 9316 \\
\hline
\end{tabular}

\section{Los modelos}

Tasas específicas de fecundidad marital

La evidencia empírica confirma que existe una marcada diferencia entre los patrones de fecundidad derivados de las tasas específicas de fecundidad marital de las poblaciones que no practican anticoncepción y aquellas en las cuales el control de la fecundidad está ampliamente difundido. Cuando en una gráfica se presentan las tasas de fecundidad marital, se observa que, en con- 
diciones de fecundidad natural, la curva es convexa y está determinada por el descenso de la fertilidad con la edad de la mujer. En contraste, en las poblaciones que controlan los nacimientos se observa que, en cuanto las parejas alcanzan el tamaño de familia deseada, las tasas específicas de fecundidad marital tienden a descender rápidamente. Por esta razón, la curva que se advierte al graficar las tasas de fecundidad marital controlada presenta una forma cóncava.

La semejanza entre las curvas de fecundidad natural y el contraste que presentan los patrones de reproducción entre poblaciones con fecundidad natural y fecundidad controlada se distingue más claramente cuando las tasas de fecundidad, a partir de 25-29 años, se expresan como una razón de las tasas en el grupo de edad 20-24. Para calcular los valores índices de las tasas específicas de fecundidad marital se considera el grupo 20-24 = 100 por ciento.

Lo anterior puede ser observado claramente en la gráfica 1, que presenta una comparación de tres poblaciones con fecundidad natural (Huteritas, 1921-1930; Crulai, 1674-1762, y Taiwan, 1900) y otras tres en las cuales el control de la natalidad es practicado en gran escala (EUA, 1960, Suecia, 1961-1965 y Bulgaria, 1969) ${ }^{3}$ Estas gráficas fueron tomadas del artículo de John Knodel (1977:219-249).

\section{Impacto de la anticoncepción}

Para evaluar el impacto de la anticoncepción en los niveles de fecundidad, Coale y Trussel (1974:185-258) han desarrollado un indice al que ellos llaman $m$ (índice de control de la fecundidad), basado en los patrones de reproducción por edad. ${ }^{4}$ Este índice $\mathrm{m}$

\footnotetext{
${ }^{3}$ Las tasas anteriores al grupo de edad 20-24 no son consideradas porque, en general, estas tasas están influidas por la edad al contraer matrimonio, concepciones premaritales y subfecundidad de la adolescencia.

${ }^{4}$ El índice m de control de la fecundidad (a partir del grupo de edad 20-24) es calculado a partir de la ecuación:$$
\mathbf{r}(\mathbf{a})=M \cdot n(a) \cdot e^{m \cdot v(a)}
$$

donde, de datos empíricos:

n(a) Fecundidad natural · promedio aritmético de 10 tablas que Henry considera como de fecundidad natural. Se descartaron las que no eran confiables.

$\mathrm{v}(a) \quad$ Función que expresa el patrón típico de control voluntario de la fecundidad. Se derivó empíricamente del comportamiento de 43 poblaciones, de la siguiente manera:
} 
Gráfica 1

Comparación de tres poblaciones con fecundidad natural y tres con fecundidad controlada

Tasas especificas de fecundidad marital

(nacimientos por mil mujeres casadas)

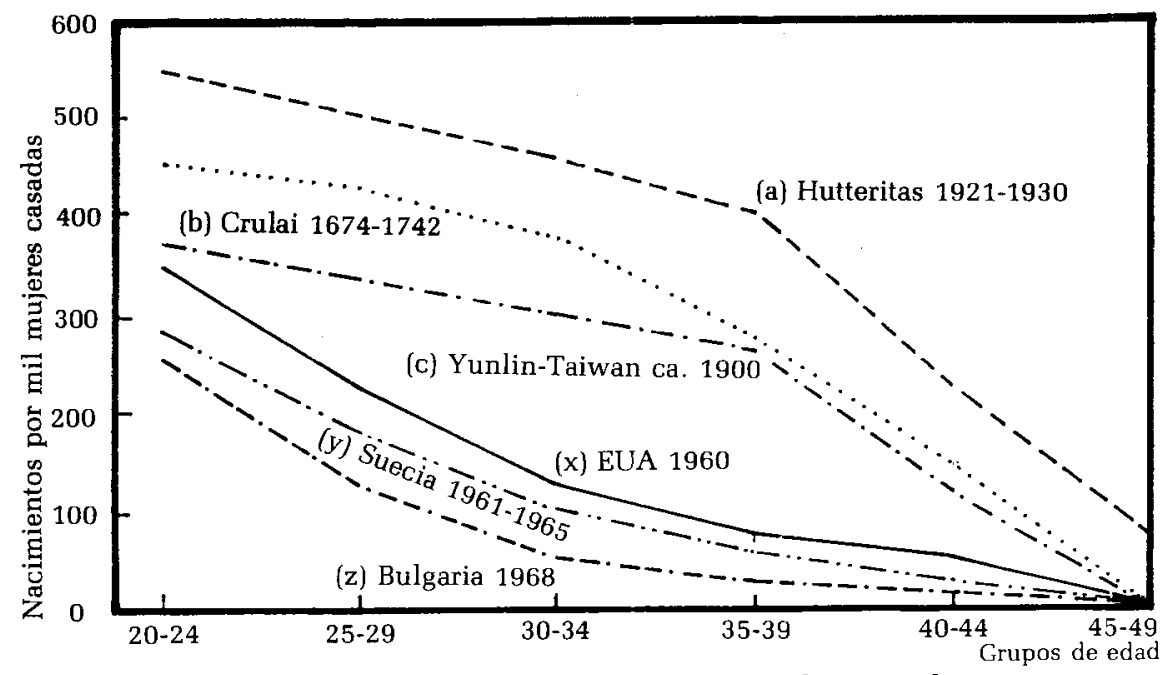

Valores índices de las tasas de fecundidad marital

( Tasa $20-24=100 \%$ )

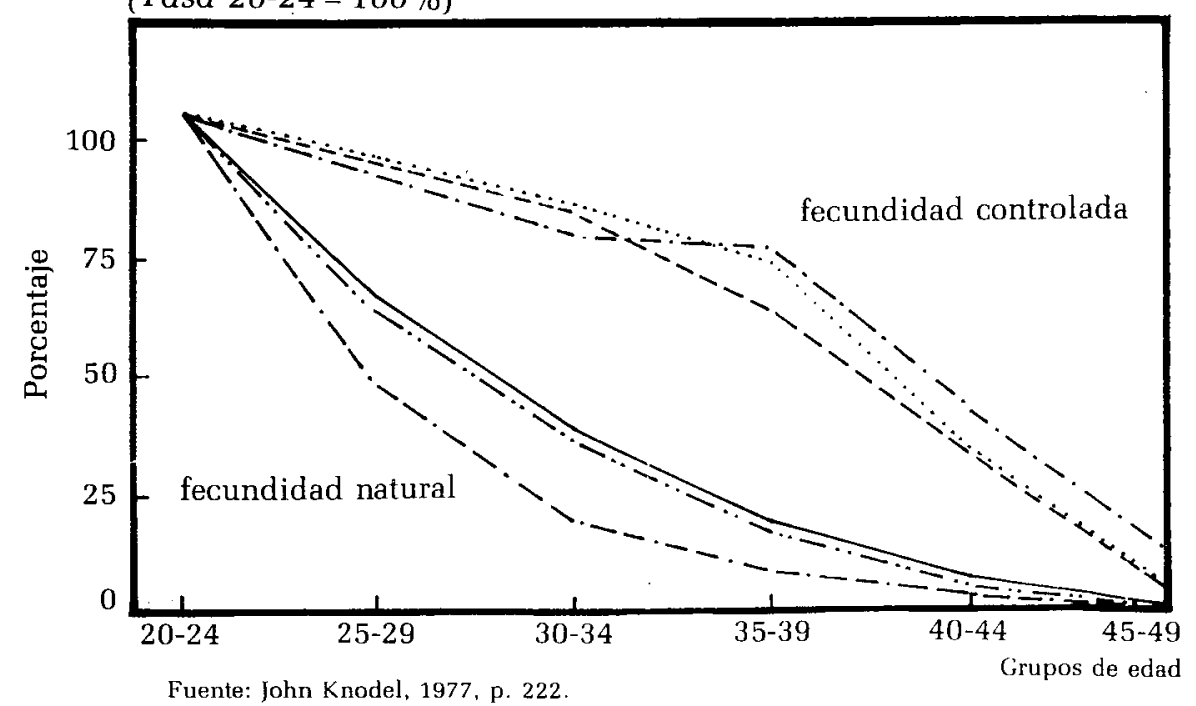


compara la distribución de fecundidad estandarizada por el nivel de edades 20-24. En esta forma su valor depende exclusivamente de la estructura por edad de la fecundidad y es independiente del nivel de fecundidad de la población. Si la forma derivada de las tasas especificas de fecundidad marital de la población que se está considerando es similar a la distribución de la fecundidad natural tipo o estándar, el valor de $\mathrm{m}$ es igual a cero en todas las edades. Por otro lado, a medida que el valor $m$ aumenta, también aumenta el grado de control de cualquier clase sobre la fecundidad. Los valores de $m$ negativos, en un determinado grupo de edad, indican que la fecundidad desciende aún más lentamente que en la distribución tipo de fecundidad natural; en otras palabras, que el nivel de la fecundidad es más alto que el del patrón típico de ausencia de control. Los valores de $m$ para las tres poblaciones de fecundidad natural -a) Huteritas 1921-1930, b) Crulai 1674-1792, c) Taiwan 1900- así como el de las tres poblaciones de fecundidad controlada - x) EUA 1960, y) Suecia 1961-1965 y z) Bulgaria 1968- se presentan en la gráfica 2.

$$
\begin{aligned}
& v(a)=\frac{1}{43} \sum_{i=1}^{43} v_{i}(a) \\
& \text { donde } v_{i}(a)=1 \mathrm{n}\left[\mathrm{r}_{i}(a) / \mathrm{M} \cdot \mathrm{n}(a)\right] \\
& M \text { es tal que } v(20-24)=0 \text {; o lo que es lo mismo } \\
& M=\frac{r(a)(20-24)}{n(a)(20-24)} \quad \frac{\text { fecundidad marital }}{\text { fecundidad natural }}
\end{aligned}
$$

La función $\mathrm{v}(\mathrm{a})$ es decreciente, lo cual indica que, al aumentar la edad, las tasas de fecundidad marital r(a) se apartan más del patrón de fecundidad natural; es decir, hay más control.

Con $\mathrm{m}=0, \mathbf{r}(a)=\mathrm{n}(a)$ : fecundidad natural. No existe control. Con $\mathrm{m}=1, \mathrm{r}(a)$ es un patrón que se desvía de la fecundidad natural como un promedio (no aritmético) de $\mathbf{4 3}$ tablas, razonablemente confiables, de principio de los años 60 , que representan un rango de diferencias en la extensión del control. A medida que $\mathrm{m}$ es más grande, mayor es la desviación respecto de la tabla de fecundidad natural, y por ende, mayor el control de la fecundidad. De (1) se puede despejar $\mathrm{m}$ : $\mathrm{m}=1 \mathrm{n}[\mathrm{r}(a) / \mathrm{M} \cdot \mathrm{n} \cdot(a)] / \mathrm{v}(a)$.

Así se puede calcular el valor de m para diferentes edades a partir de una tabla emp/rica de fecundidad marital $\mathbf{r}(a)$.

Entre las poblaciones que controlan la fecundidad, los valores de $\mathrm{m}$ no son completamente uniformes para todas las edades, lo que significa que el patrón de control no es del todo válido para algunas poblaciones particulares.

$$
\begin{aligned}
& \mathrm{r}(a)=M \cdot n(a) \mathrm{e}^{m \cdot v(a)} \\
& \frac{r(a)=e^{m \cdot v(a)}}{M \cdot n(a)} \quad \frac{r[a)}{M \cdot n(a)}=e^{m \cdot v(a)}
\end{aligned}
$$




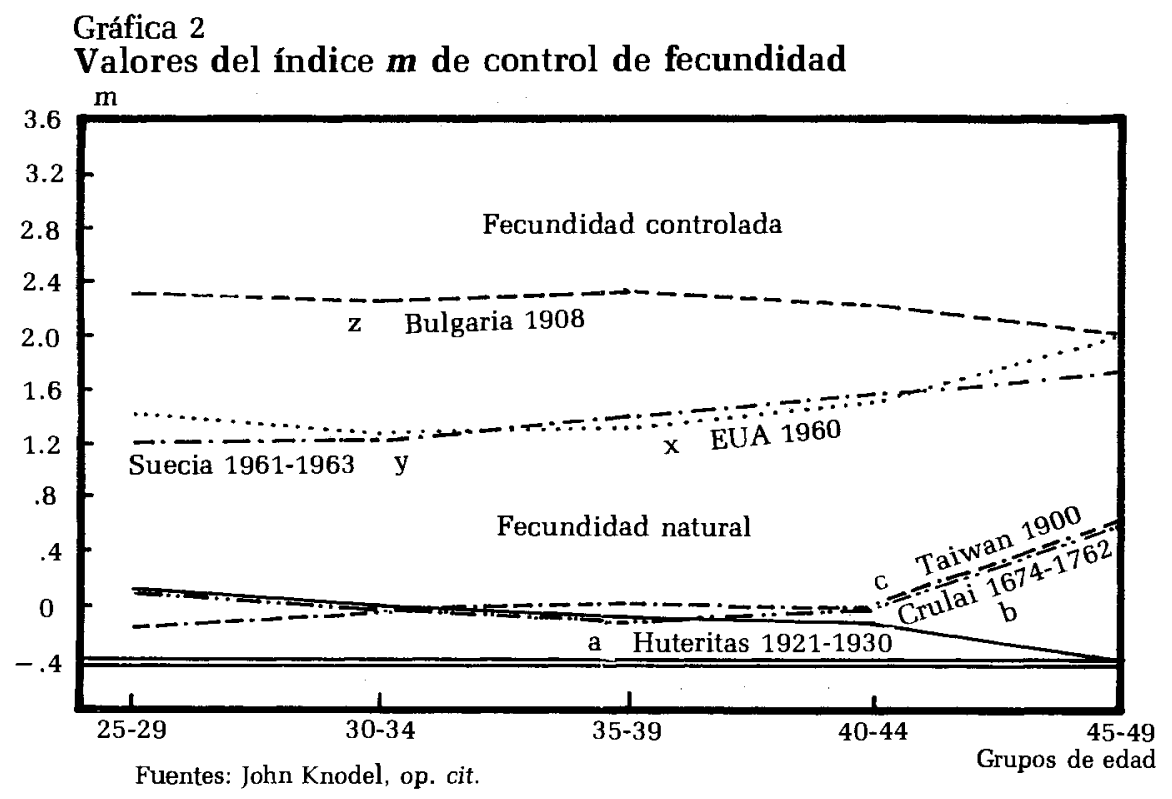

4. Evolución de la fecundidad y de la anticoncepción marital en las zonas rurales y urbanas de México, 1969-1987

A continuación se presentan separadamente para las zonas rurales, las urbanas y el total del país, los principales resultados derivados de todas las encuestas en el estudio. Para tener una mejor apreciación de lo que se describe, aparecen el cuadro 2 , con los valores de las tasas específicas de fecundidad marital, y el cuadro 3 , con los del índice $m$ de control de nacimientos. Para facilitar la comparación entre las diferentes encuestas a través del tiempo,

$$
\begin{aligned}
& \text { in } \frac{r(a)}{M \cdot n(a)}=m \cdot v \cdot(a) \\
& \operatorname{in} \frac{\frac{r(a)}{M \cdot n(a)}}{v(a)}=m_{m=1 n[r(a) / M \cdot n(a)] / v(a)} \\
& \begin{array}{ccccccc} 
& 20-24 & 25-29 & 30-34 & 35-39 & 40-44 & 45-49 \\
n(a) & 0.4597 & 0.4309 & 0.3946 & 0.3223 & 0.1671 & 0.237 \\
v(a) & 0.0000 & -0.279 & -0.677 & -1.047 & -1.414 & -1.671
\end{array}
\end{aligned}
$$


CUADRO 2

Evolución de la fecundidad en México, 1970-1982.

Tasas específicas de fecundidad marital

\begin{tabular}{|c|c|c|c|c|c|c|}
\hline Grupos de Edad & $1970^{1}$ & $1976^{2}$ & $1979^{3}$ & $1981^{4}$ & $1982^{5}$ & $1987^{6}$ \\
\hline \multicolumn{7}{|c|}{ Total } \\
\hline $15-19$ & & 0.506 & 0.391 & & 0.424 & 0.264 \\
\hline $20-14$ & & 0.456 & 0.362 & & 0.332 & 0.314 \\
\hline $25-29$ & & 0.305 & 0.266 & & 0.245 & 0.258 \\
\hline $30-34$ & & 0.251 & 0.228 & & 0.188 & 0.172 \\
\hline $35-39$ & & 0.201 & 0.159 & & 0.125 & 0.125 \\
\hline $40-44$ & & 0.065 & 0.071 & & 0.051 & 0.036 \\
\hline $45-49$ & & 0.024 & 0.008 & & 0.016 & 0.005 \\
\hline$\underline{\text { TGF }}$ & & 9.04 & 7.43 & & 6.90 & 5.85 \\
\hline \multicolumn{7}{|c|}{ Urbana } \\
\hline $15-19$ & & 0.473 & 0.408 & & 0.431 & 0.247 \\
\hline $20-24$ & & 0.427 & 0.328 & & 0.327 & 0.295 \\
\hline $25-29$ & & 0.268 & 0.232 & & 0.229 & 0.214 \\
\hline $30-34$ & & 0.179 & 0.179 & & 0.158 & 0.136 \\
\hline $35-39$ & & 0.119 & 0.094 & & 0.091 & 0.084 \\
\hline $40-44$ & & 0.062 & 0.057 & & 0.032 & 0.030 \\
\hline $45-49$ & & 0.016 & 0.004 & & 0.010 & 0.001 \\
\hline TGF & & 7.72 & 6.51 & & 6.39 & 5.03 \\
\hline \multicolumn{7}{|c|}{ Rural } \\
\hline $15-19$ & 0.442 & 0.518 & 0.380 & 0.424 & 0.419 & 0.280 \\
\hline $20-24$ & 0.456 & 0.467 & 0.394 & 0.403 & 0.338 & 0.337 \\
\hline $25-29$ & 0.422 & 0.340 & 0.300 & 0.296 & 0.261 & 0.316 \\
\hline $30-34$ & 0.354 & 0.311 & 0.285 & 0.253 & 0.217 & 0.221 \\
\hline $35-39$ & 0.273 & 0.264 & 0.217 & 0.207 & 0.155 & 0.170 \\
\hline $40-44$ & 0.137 & 0.067 & 0.087 & 0.096 & 0.071 & 0.044 \\
\hline $45-49$ & 0.038 & 0.031 & 0.013 & 0.011 & 0.022 & 0.012 \\
\hline TGE & 10.610 & 9.99 & 8.38 & 8.45 & 7.41 & 6.84 \\
\hline
\end{tabular}

Fuentes: ${ }^{1}$ Encuesta Rural de Fecundidad, 1970. (Localidades menores de 20000 habitantes); ${ }^{2}$ Encuesta Mexicana de Fecundidad, 1976; ${ }^{3}$ Encuesta Nacional de Prevalencia en 1978 y 1979. El uso de métodos anticonceptivos; ${ }^{4}$ Encuesta Rural de Planificación Familiar 1981 (localidades de menos de 2500 habitantes); ${ }^{5}$ Encuesta Nacional Demográfica, 1982, Fátima Juárez, El Colegio de México. Periodo de referencia (36 meses previos a la fecha de la encuesta); ${ }^{6}$ Encuesta Nacional sobre Fecundidad y Salud, 1987. (Promedio de los años 1984-1986).

en la misma forma se presentan las gráficas alusivas a las cifras anteriores y que se describen a continuación: gráficas 3,5 y 7 tasas de fecundidad marital rural, urbana y total respectivamente. Gráficas 4, 6 y 8 valores del índice $m$ de control de fecundidad rural, urbana y total. 
CUADRO 3

Evolución de la fecundidad en México, 1970-1982. Índices $m$ de control de la fecundidad

\begin{tabular}{|c|c|c|c|c|c|c|}
\hline Grupos de Edad & 1970 & 1976 & 1979 & 1981 & 1982 & $1987^{*}$ \\
\hline \multicolumn{7}{|c|}{ Total } \\
\hline \multicolumn{7}{|l|}{$20-24$} \\
\hline $25-29$ & & 1.156 & 0.871 & & 0.859 & 0.107 \\
\hline $30-34$ & & 0.639 & 0.458 & & 0.618 & 0.398 \\
\hline $35-39$ & & 0.431 & 0.447 & & 0.600 & 0.314 \\
\hline $40-44$ & & 0.653 & 0.435 & & 0.609 & 0.723 \\
\hline $45-49$ & & -0.023 & 0.514 & & 0.044 & 0.229 \\
\hline \multicolumn{7}{|c|}{ Urbana } \\
\hline \multicolumn{7}{|l|}{$20-24$} \\
\hline $25-29$ & & 1.442 & 1.008 & & 1.040 & 0.917 \\
\hline $30-34$ & & 1.061 & 0.670 & & 0.054 & 0.919 \\
\hline $35-39$ & & 0.883 & 0.857 & & 0.891 & 0.863 \\
\hline $40-44$ & & 0.649 & 0.521 & & 0.919 & 0.900 \\
\hline $45-49$ & & 0.185 & 0.870 & & 0.345 & 1.636 \\
\hline \multicolumn{7}{|c|}{ Rural } \\
\hline \multicolumn{7}{|l|}{$20-24$} \\
\hline $25-29$ & -0.349 & 0.907 & 0.744 & 0.868 & 0.695 & 0.471 \\
\hline $30-34$ & 0.028 & 0.376 & 0.253 & 0.460 & 0.427 & 0.664 \\
\hline $35-39$ & 0.068 & 0.206 & 0.230 & 0.294 & 0.408 & 0.542 \\
\hline $40-44$ & 0.212 & 0.658 & 0.352 & 0.297 & 0.382 & 0.815 \\
\hline $45-49$ & -0.137 & -0.141 & 0.274 & 0.382 & -0.136 & 0.710 \\
\hline
\end{tabular}

* Para 1987 se utilizó el promedio de los años 1984-1986, excepto para el total del grupo de edad 25-29 en el cual se utilizó el promedio de 1982-1986

Fuentes: Cálculos obtenidos con la información del cuadro 3.

\section{Zonas rurales ${ }^{5}$}

Al analizar los resultados de la más distante encuesta que se incluye en este estudio (FERU, 69-70) y que aparecen en los cuadros 2 y 3 (primera columna) así como en las gráficas 3 y 4 , observamos que la curva que presentan las tasas específicas de fecundidad es convexa, o sea, equivalente a la forma típica que presentan las poblaciones en las que la práctica de la anticoncepción

${ }^{5}$ Cabe resaltar el hecho de que hubo dos encuestas: 1969-1970 y 1981 que aunque con diferentes criterios para definir el contexto urbano-rural, fueron dirigidas específicamente a las zonas rurales del país. Este hecho debe tenerse en cuenta al observar los resultados. Por la razón expresada anteriormente, para las áreas rurales se presenta mayor información que para las urbanas y para el total del país. 
no es significativa. Por otra parte, los valores del índice m de control de fecundidad (gráfica 4) están muy cercanos a cero. De los 30 a los 44 años se registra un ligero control que, sin duda, está relacionado con el limitado número de mujeres que reportaron haber hecho uso de la anticoncepción. En consecuencia podemos inferir que en 1969, en las áreas rurales del país, el patrón por edad era muy cercano al que se presenta en las condiciones de fecundidad natural. En estas fechas la tasa global de fecundidad era de 10.6, o sea un promedio de casi 11 hijos por mujer casada (cuadro 2, primera columna).

A partir de entonces las curvas que forman las tasas maritales de las otras encuestas poco a poco van perdiendo la apariencia convexa hasta llegar a formar una línea más o menos recta en la encuesta de 1982. El proceso de aumento en la anticoncepción a través del tiempo se observa claramente en la gráfica 4, en la cual, a medida que las fechas del levantamiento de las encuestas son más recientes, el índice $\mathrm{m}$ se aleja cada vez más de cero. La excepzión se presenta en el grupo de 45-49 años, ${ }^{6}$ el cual presenta valores negativos en las encuestas realizadas en 1969, 1976 y 1982. Esto implica niveles de fecundidad aún más altos que en las poblaciones "tipo" de "fecundidad natural"'. Este hecho, aunque no afecta en gran medida la fecundidad total, puede tener varias explicaciones, entre ellas: a) que en las áreas rurales del país algunas mujeres que están al final de su etapa reproductiva no aceptan o no tienen acceso a la anticoncepción y siguen teniendo hiios; b) también podría deberse a errores diversos en la captación de los datos o bien del diseño de la muestra, y c) las fuentes de información no son estrictamente comparables. En lo que respecta a la comparabilidad, es necesario recordar que la ERPF (1981) fue Jirigida únicamente a las localidades más rurales del país (menos de 2500 habitantes). Esto la hace no ser comparable con las demás.

Volviendo al cuadro 2, podemos observar que de 1970 a 1982 a tasa global de fecundidad marital descendió en 3.2 (de 10.6 a 7.4 hijos), o sea un equivalente a $30 \%$ en doce años, que significa $2.5 \%$ anual. Por otra parte, durante los cinco años comprendidos mtre 1982 y 1987 el descenso sólo fue de 0.6 (de 7.4 a 6.8 hijos), sorrespondiente a sólo $1.6 \%$ anual. En otras palabras, aun cuando ıl descenso continuó, su ritmo disminuyó.

\footnotetext{
${ }^{6}$ La fecundidad marital después de los 45 años de edad es poco confiable y las cifras se deben tomar con precaución ya que, por una parte, las mujeres están al final de su periodo reproductivo y, por otra, el reducido número de casos en эse grupo de edad puede no ser representativo de toda la población.
} 
Gráfica 3

A. Tasas de fecundidad marital.

Nacim. mil mujeres casadas (rural)

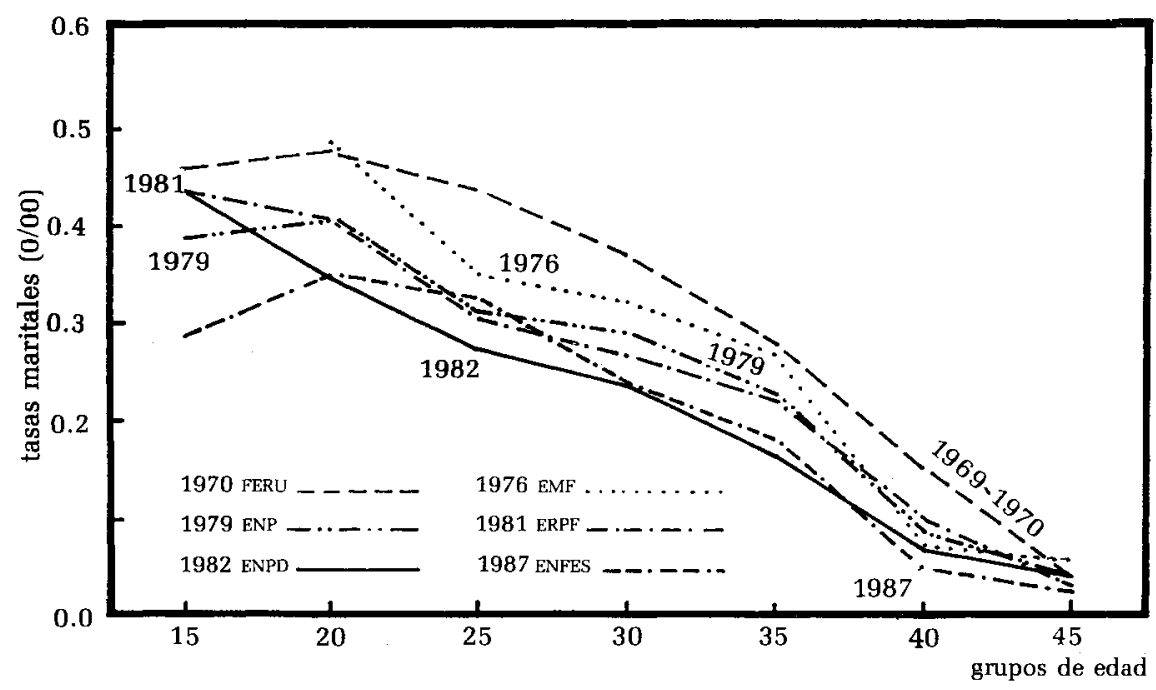

Gráfica 4

C. valores del índice m de control de fecundidad (rural)

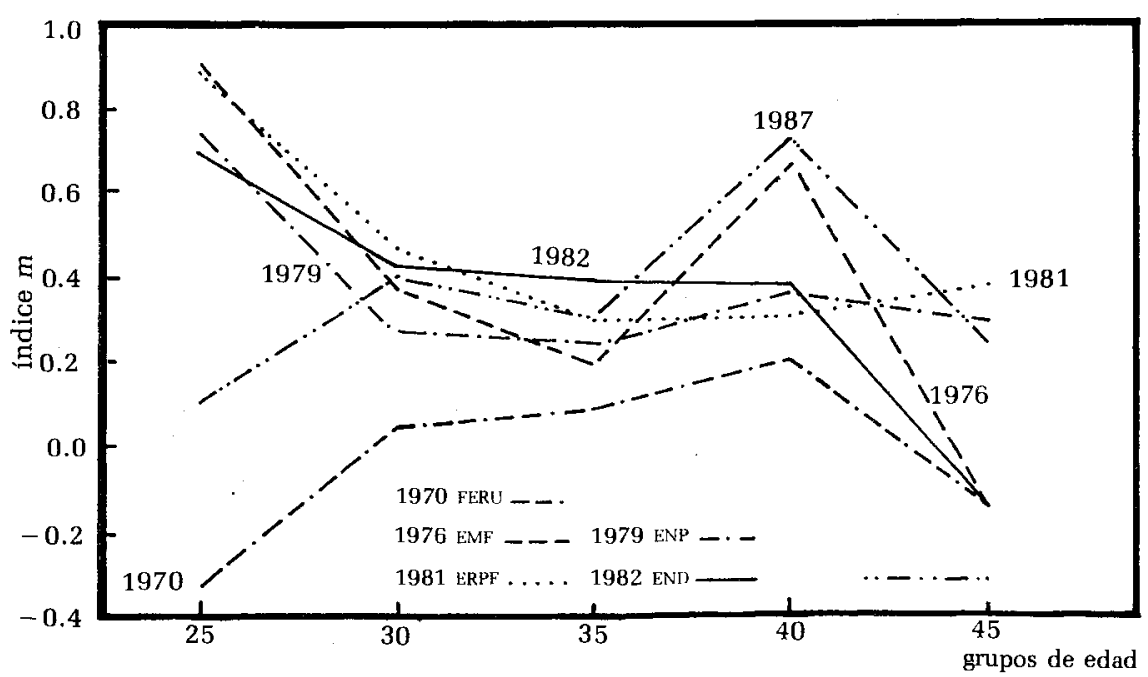


Gráfica 5

A. tasas de fecundidad marital.

Nacim. mil mujeres casadas (urbana)

as maritales $(0 / 00)$

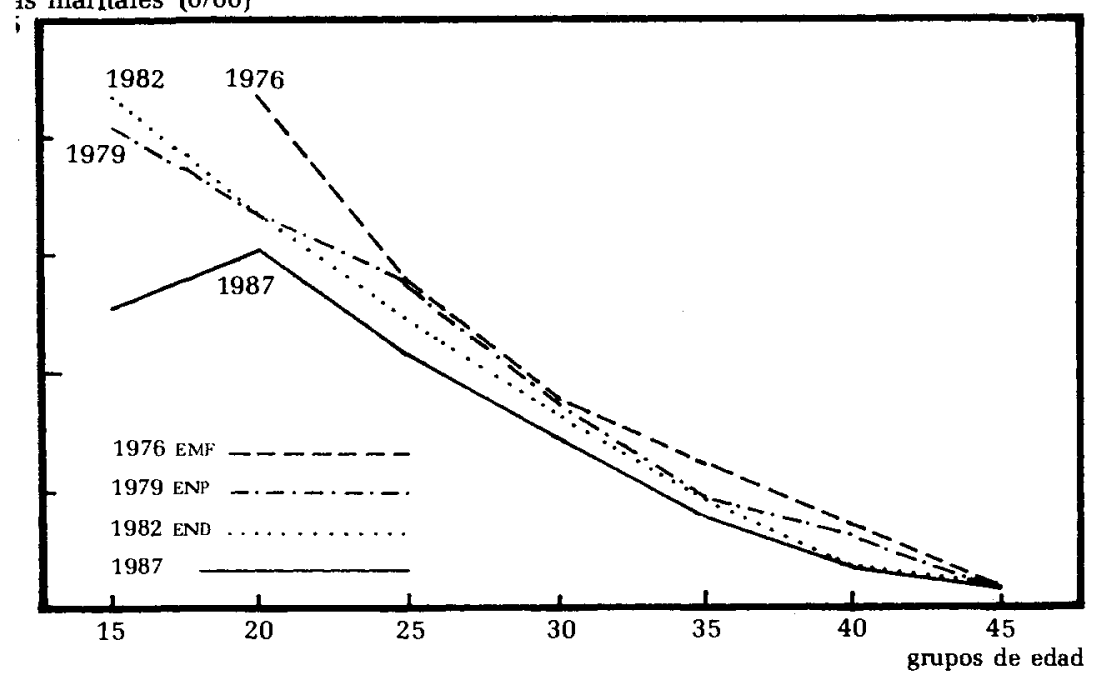

Gráfica 6

C. valores del índice m de control de fecundidad (urbana)

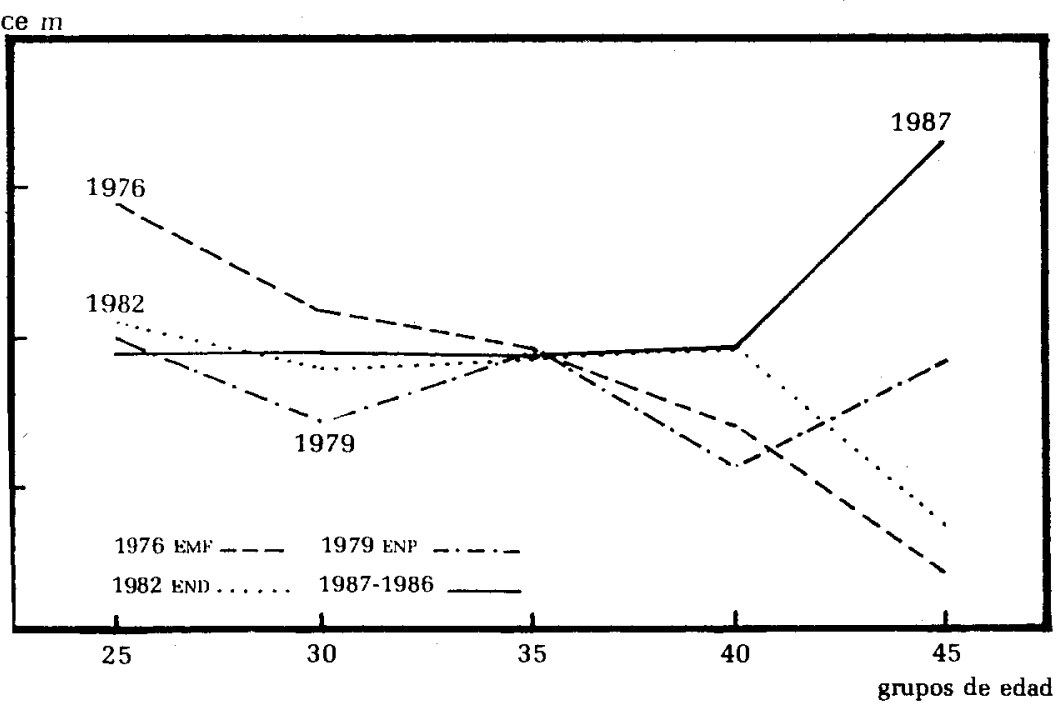


Gráfica 7

A. tasas de fecundidad marital. Nacim. mil mujeres casadas (total)

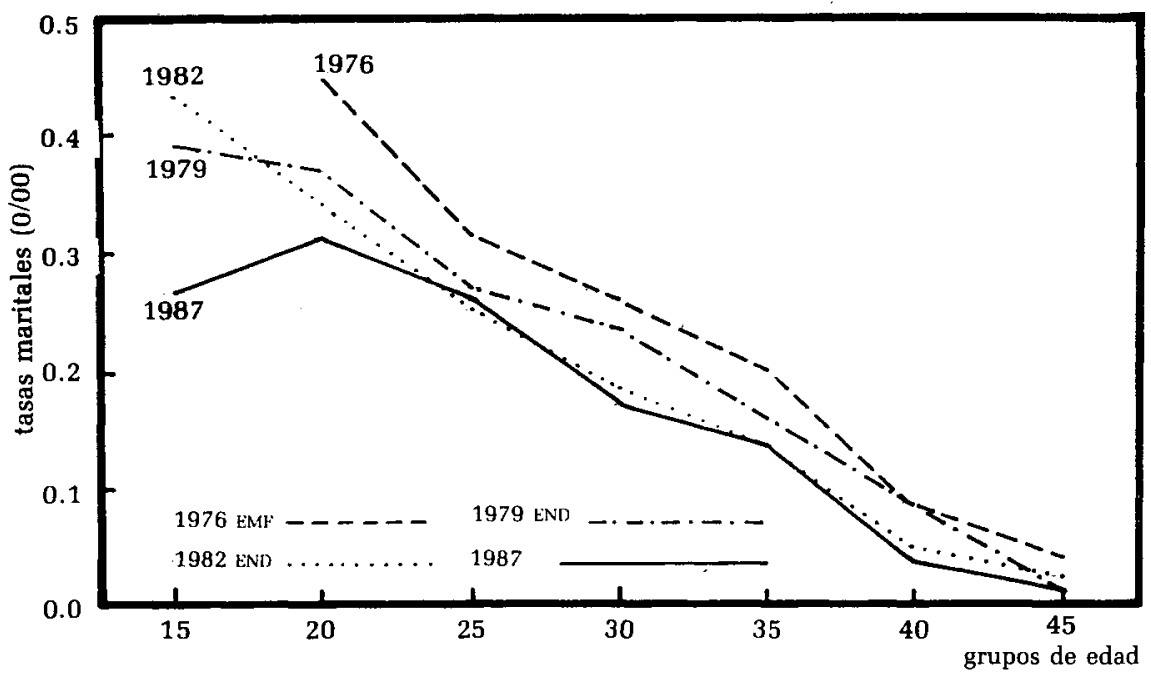

Gráfica 8

C. valores del índice $\boldsymbol{m}$ de control de fecundidad (total)

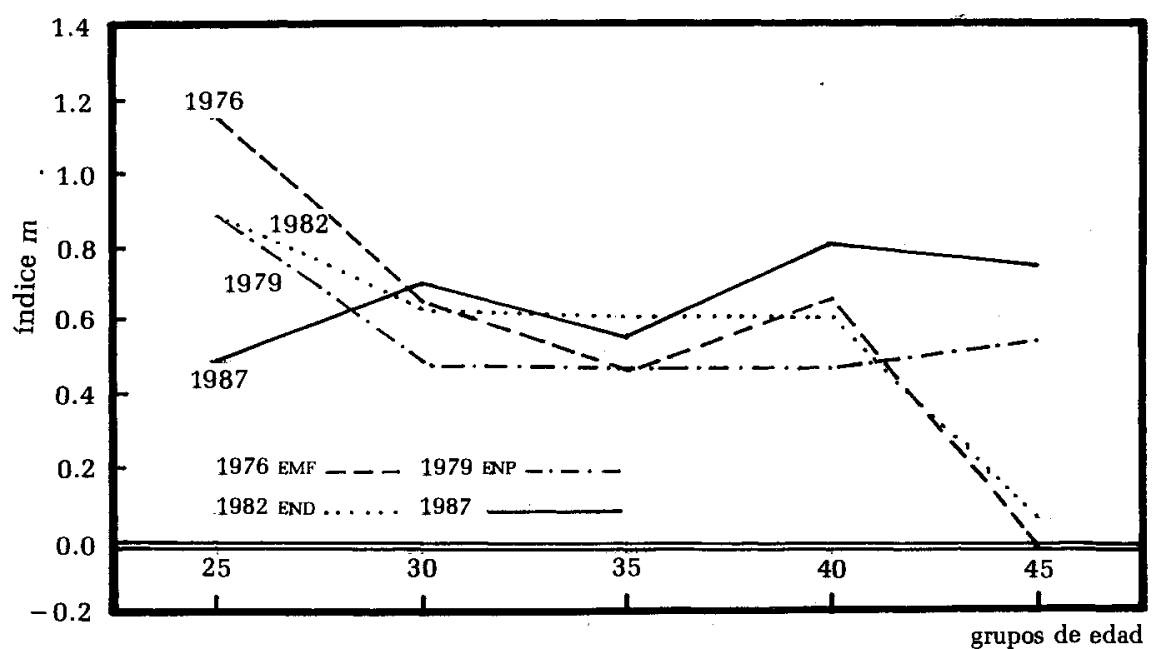




\section{Zonas urbanas}

Al analizar la fecundidad en las áreas urbanas (cuadros 2 y 3 , segundo bloque y gráficas 5 y 6) el panorama que se nos presenta es diferen te del de las áreas rurales. Aunque la curva que forman las tasas maritales no es totalmente cóncava como la que presentan las poblaciones donde la anticoncepción es ampliamente practicada, sí se observa cierta tendencia hacia ese estado. Por lo que respecta a los índices m es fácil visualizar que en todas las encuestas y en todos los grupos de edad se registra, en mayor o menor grado, el impacto de la anticoncepción, ya que todos los valores están arriba de cero. Llama la atención el hecho de que las mujeres mayores de 45 años de edad en las encuestas realizadas en 1976, 1979 y 1982 presentaban niveles moderados de anticoncepción. Sin embargo, en 1987 presentan el índice más alto. A este respecto podría especularse que en las áreas urbanas las mujeres que se encuentran al final de su etapa reproductiva ${ }^{\gamma}$ ya no desean tener más hijos y el tiempo de actividad reproductiva se está concentrando entre los 25 y los 40 años.

Otro punto importante respecto al grado de control en las áreas urbanas es que en el grupo 35-39 los valores de m en las 4 encuestas son más o menos constantes y muy cercanos entre sí (gráfica 6), lo cual podría ind icar que el cambio en el uso de anticonceptivos se ha concentrado en las demás edades. Por otro lado, salvo en el grupo 45-49, como ya se dijo antes, muestra un comportamiento irregular, las diferencias 1982-1987 en todos los grupos, son mínimas. Lo anterior significa que, durante ese periodo, disminuyó el ritmo del descenso de la fecundidad en las áreas urbanas.

El diferencial rural-urbano en el descenso de la fecundidad en los periodos 1976-1982 y 1982-1987

Los hallazgos anteriores indican que mientras en el periodo 19761982 en las áreas rurales aumentaron las prácticas anticonceptivas en forma considerable, en las urbanas las ganancias fueron mucho menos importantes. En consecuencia, la contribución de las áreas rurales al ritmo del descenso de la fecundidad fue mayor que la de las áreas urbanas. Esto se comprueba al obtener la diferencia de las tasas en el periodo 1976-1982, que registró para las áreas

\footnotetext{
${ }^{7}$ En los países desarrollados en general, a partir de los 45 años no se calculan tasas de fecundidad porque, o ya no ocurren, o bien es escaso el número de nacimientos.
} 
urbanas un descenso de 1.3 hijos, o sea $17.2 \%$; mientras que, para las rurales, fue de 2.5 hijos, que es el equivalente a $25.8 \%$ menos. Sin embargo, este hecho no permaneció, ya que entre 1982 y 1987 se observa lo inverso. Es decir, en este último periodo, se registró un descenso de 1.4 hijos, o sea, $21.3 \%$ en las áres urbanas, y de 0.6 o el equivalente a $8 \%$ en las rurales. Este hecho inusitado nos hace pensar que en México el descenso de la fecundidad no ha sido proporcional ni constante, sino que ha variado según diferentes condiciones individuales y sociales difíciles de detectar con el tipo de información que manejamos.

\section{Total del país}

Al conjuntar el comportamiento urbano y rural para observar la fecundidad en todo el país (cuadros 2 y 3 ; gráficas 7 y 8 ) se perciben algunos cambios acentuados propios de una etapa de transición. En las gráficas citadas.se percibe cierta tendencia a la concavidad. Lo anterior se corrobora en los índices $m$ que, aunque muestran ciertas oscilaciones, definitivamente la práctica de la anticoncepción se acentúa a través del tiempo. Conforme a los datos del cuadro 2, el descenso total en el país entre 1976 y 1987 fue de 3.2 hijos, equivalente a 31.5 por ciento.

Hasta aquí hemos visto claro lo siguiente: a) que durante el periodo de observación se perdieron las condiciones de fecundidad natural en las áreas rurales del país y, a través del tiempo, los descensos en la fecundidad y consecuentemente los ascensos en el uso de anticonceptivos se van acentuando; b) que en las áreas urbanas la anticoncepción es ampliamente practicada y su uso se extiende cada vez más, y c) que, al menos en algunas ocasiones, el ritmo de aumento en la limitación del tamaño de la familia ha sido mayor en las áreas rurales que en las urbanas.

\section{Consideración final a manera de conclusión}

Algunos autores declaran que el descenso de la fecundidad en los países desarrollados se atribuye a factores asociados con el proceso de modernización. Conforme a este enfoque tradicional, en México se han llevado a cabo varios estudios en los que se ratifica la existencia de una relación inversa entre el nivel de fecundidad y la educación, el tamaño de la localidad, la mortalidad infantil y otros.

Aunque no se puede dejar de reconocer que una menor fecun- 
didad por lo general está ligada a mejores condiciones socioeconómicas, al observar el comportamiento del descenso registrado en este estudio en las zonas rurales del país tanto en la encuesta de 1981 como en el periodo 1976-1982 (véanse las gráficas 9 y 10), la teoría de la modernización pierde fuerza. Sin embargo, lo observado en el quinquenio posterior (1982-1987) nos hace reconsiderar sobre esta afirmación (véanse el cuadro 4 y la gráfica 11).

\section{Gráfica 9}

Descensos en las tasas de fecundidad marital 1976-1982 en términos absolutos

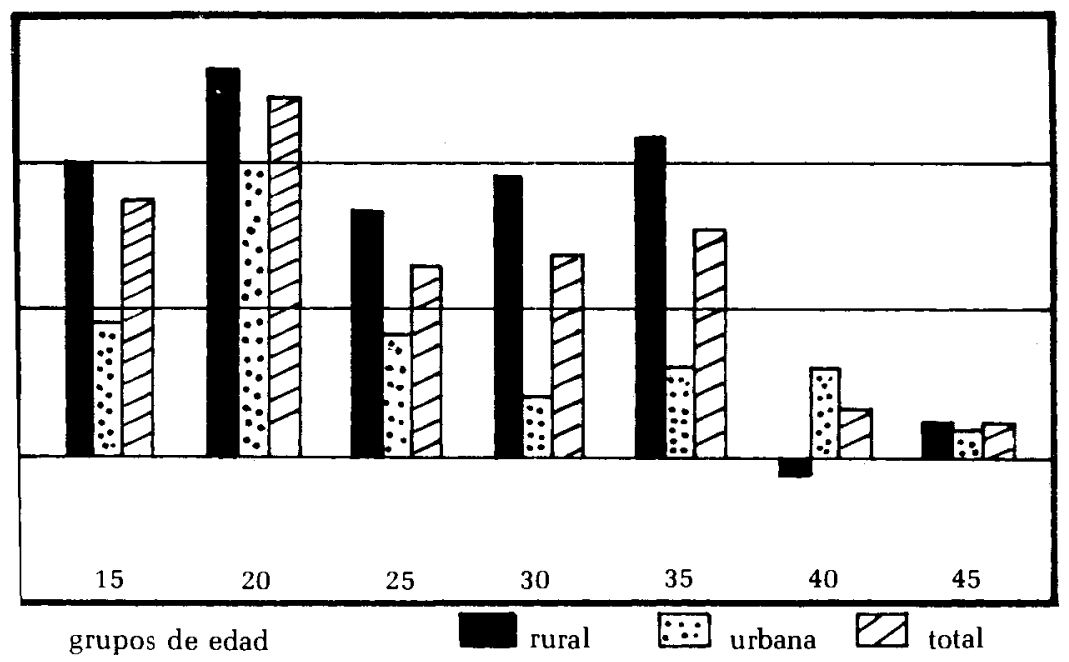

Por una parte, la diseminación de los métodos anticonceptios y la extensa propaganda por radio y televisión han ayudado disminuir los "costos" tanto objetivos como subjetivos del ontrol de la fecundidad. Por la otra, no se han registrado cambios ocioeconómicos sustanciales en las zonas menos privilegiadas el país; esto es, las áreas rurales, de modo que esta situación proablemente responde a las oscilaciones propias de las etapas de ransición.

La hipótesis inicial de este trabajo era ver si la anticoncepción n las zonas rurales del país podría practicarse significativamente

${ }^{8}$ El término "costos" de la anticoncepción, además del costo monetario del nticonceptivo, incluye, entre otros, los costos de uso, el acceso, la disponibiliad, el sentido de culpa, los efectos colaterales y demás inconvenientes que acaree su práctica. 
CUADRO 4

Descensos en las tasas específicas de fecundidad marital.

Diferencia entre las tasas 1976-1982

(valores absolutos)

\begin{tabular}{lccl}
\hline & Rural & Urbana & Total \\
\hline $15-19$ & 0.099 & 0.042 & 0.082 \\
$20-24$ & 0.129 & 0.110 & 0.124 \\
$25-29$ & 0.044 & 0.039 & 0.060 \\
$30-34$ & 0.094 & 0.021 & 0.063 \\
$35-39$ & 0.109 & 0.028 & 0.076 \\
$40-44$ & +0.004 & 0.030 & 0.014 \\
$45-49$ & 0.018 & 0.006 & 0.008 \\
En la TGFM & 2.58 & 1.33 & 2.14 \\
\hline
\end{tabular}

Porcentajes de los descensos en las tasas de fecundidad marital 1976-1982

\begin{tabular}{|c|c|c|c|}
\hline $15-19$ & 19.1 & 8.9 & 16.2 \\
\hline $20-24$ & 13.7 & 23.4 & 27.2 \\
\hline $25-29$ & 23.2 & 14.6 & 19.7 \\
\hline $30-34$ & 30.2 & 11.7 & 25.1 \\
\hline $35-39$ & 31.3 & 23.5 & 37.8 \\
\hline $40-44$ & +6.0 & 48.4 & 21,5 \\
\hline $45-49$ & 25.9 & 37.5 & 33.3 \\
\hline En la TGFM & 25.8 & 17.2 & 23.7 \\
\hline \multicolumn{4}{|c|}{ Descensos en las tasas de fecundidad marital 1982-1987 } \\
\hline $15-19$ & 0.139 & 0.184 & 0.16 \\
\hline $20-24$ & 0.066 & 0.032 & 0.018 \\
\hline $25-29$ & -0.055 & 0.015 & -0.013 \\
\hline $30-34$ & -0.004 & 0.022 & 0.016 \\
\hline $35-39$ & -0.015 & 0.007 & 0.000 \\
\hline $40-44$ & 0.027 & 0.002 & 0.015 \\
\hline $45-49$ & 0.010 & 0.009 & 0.011 \\
\hline En la TGFM & 0.57 & 1.36 & 1.05 \\
\hline \multicolumn{4}{|c|}{ Porcentajes de descensos 1982-1987 } \\
\hline $15-19$ & -33.2 & 42.7 & 37.7 \\
\hline $20-24$ & -0.3 & 9.8 & 5.4 \\
\hline $25-29$ & +21.1 & 6.6 & -5.3 \\
\hline $30-34$ & +1.8 & 13.9 & 8.5 \\
\hline $35-39$ & +9.7 & 7.7 & 0.0 \\
\hline $40-44$ & -38.0 & 6.3 & 29.4 \\
\hline $45-49$ & -45.5 & 90.0 & 68.8 \\
\hline En la TGFM & -7.7 & 21.3 & 15.2 \\
\hline
\end{tabular}


Gráfica 10

Descensos en las tasas de fecundidad marital 1976-1982 en términos relativos

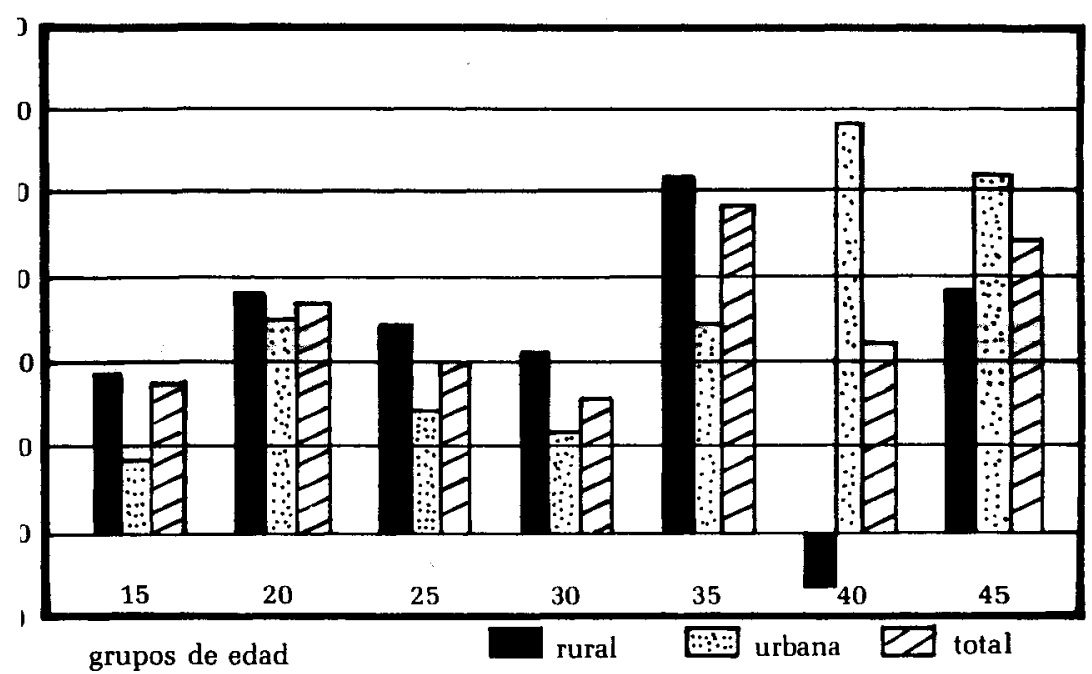

Gráfica 11

Descensos en las tasas de fecundidad marital 1976-1982 en términos absolutos

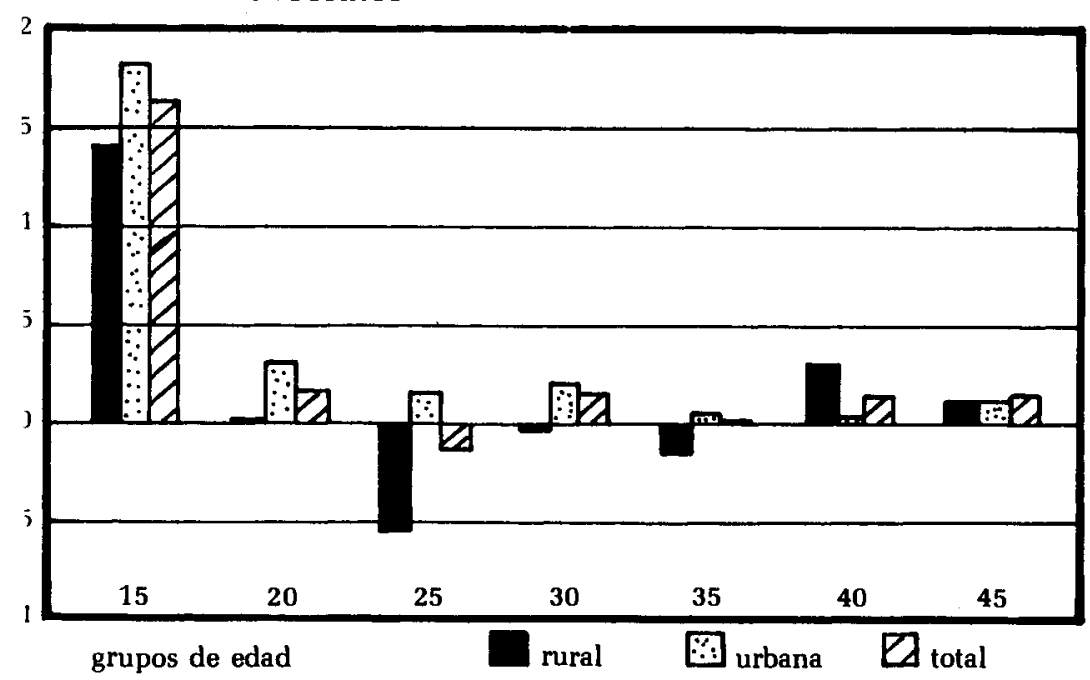


Gráfica 12

Descensos en las tasas de fecundidad marital 1976-1982 en términos relativos

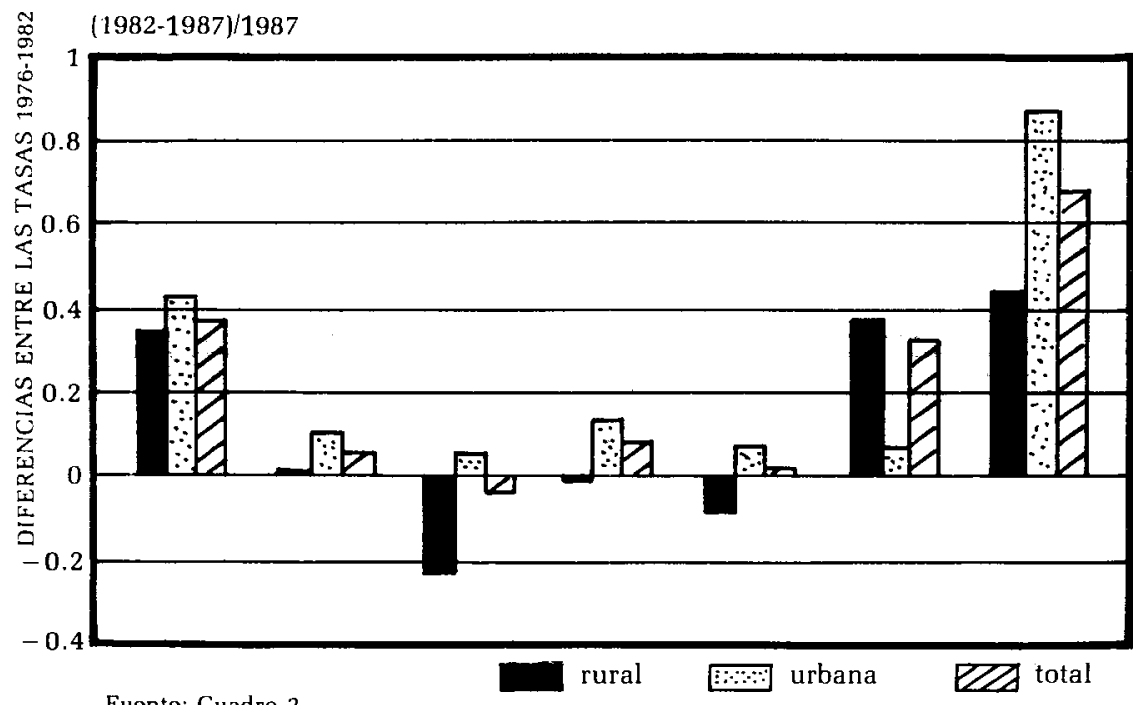

Fuente: Cuadro 2

aún con un desarrollo socioeconómico incipiente. Hemos visto que este postulado se confirma hasta cierto punto, pero que es probable que un descenso sostenido de la fecundidad en las zonas más deprimidas del país sólo pueda lograrse con cambios socioeconómicos sustantivos.

\section{Bibliografía}

Bronfman, Mario, Elsa López y Rodolfo Tuirán, 1986. "Práctica Anticonceptiva y clases sociales en México. La experiencia reciente". Estudios Demográficos y Urbanos, vol. 1, núm. 2, pp. 165-203.

Bulatao, Rodolfo A. y Lee Ronald D., 1983. "Determinants of Fertility in Developing Countries", vol. I, Supply and Demand for Children, Studies in Population, Academic Press.

Centro Latinoamericano de Demografía (Celade), 1970. Programa de Encuestas Comparativas de Fecundidad en América Latina, México Rural.

, 1980. La fecundidad en México. Estimación de niveles y tendencias, 1950-1979, Santiago de Chile.

Coale, Ansley, J., 1973. "Demographic Transition", international Population Conference, Lieja, vol. I, Bélgica. , y T. James Trussell, 1974. "Model Fertility Schedules: Varia- 
tions in the Age Structure of childbearing In Human Populations" Population Index 40, pp. 185-258.

Consejo Latinoamericano de Ciencias Sociales, 1985. Reproducción de la Población y Desarrollo, núm. 5, São Paulo.

Consejo Nacional de Población (Conapo), Resultados Principales de la Encuesta Nacional Demográfica de 1982.

Coordinación del Programa Nacional de Planificación Familiar (CPNPF), Encuesta nacional de prevalencia en el uso de métodos anticonceptivos con módulo fecundidad/mortalidad, México.

Coordinación del Programa Nacional de Planificación Familiar (CPNPF), Encuesta Nacional de Prevalencia en el uso de métodos anticonceptivos, resultados nacionales, México, 1980.

Dirección General de Estadística (DGE), 1979. Encuesta mexicana de Fecundidad, 3 vols., Coordinación del Sistema Nacional de Información, sPP, México. Coordinación del Programa de Planificación Familiar (CPNPE), Encuesta Nacional de prevalencia en el uso de métodos anticonceptivos, resultados nacionales, México.

Easterlin, Richard A., 1969. "A Socieconomic Theory of fertility". A Survey of Economic Factors in American Fertility" en S.J. Berh man, Leslie Corda y Ronald Freedman (comps.), Fertlity and Family Planning: A World View. Ann Arbor: University of Michigan Press.

García, Brígida, 1976. "Anticoncepción en el México rural, 1969" Demografía y Economía, El Colegio de México, vol. x, núm. 3, pp. 297350.

García y Garma, Irma Olaya, 1980. Fertility Determinants in rural Mexico, tesis doctoral. Universidad de Pensylvania.

"Estado del conocimiento relativo a los determinantes de los niveles de fecundidad en México", en Beatriz Figueroa (comp.), 1989. La fecundidad en México: cambios y perspectivas, CEDDU, el Colegio de México.

Preludio del descenso de la fecundidad en México, Consejo Latino de Ciencias Sociales. Reproducción de la población y desarrollo, Clacso 5, pp. 133-155.

Instituto Mexicano del Seguro Social (IMSS), 1983. Encuesta rural de planificación familiar, 1981. Resultados Nacionales.

Jefatura de Servicios de Planificación Familiar. Encuesta Rural de Planificación Familiar (ERPF), IMSS, 1981.

Knodel, John, 1977. "Family Limitation and the Fertility Transition: Evidence from the Age Patterns of Fertility in Europe and Asia", Population Studies 31, 2, pp. 219-249.

Nuñez Fernández, Leopoldo, México: las encuestas nacionales en la estimación de los niveles de fecundidad. Jefatura de Servicios de Planificación Familiar, IMSS.

Ordorica Manuel, 1981. "Evaluation of the Mexican Fertility Surveys 1976-1977”, MFS Scientific Report, núm. 21. International Statistical Institute, Londres.

?otter, Joseph E. "Problems in Using Birth history analysis to estimate trens in fertility", Population Studies 31.2. 
Population Reference Bureau, Inc. World Population Data Sheet, varios años.

Quilodrán, Julieta, 1979. "La nupcialidad en las áreas rurales de México",

Demografía y Economía, 13, núm. 3, pp. 263-316.

Quilodrán, Julieta, 1982. Impacto de la disolución de uniones sobre la fecundidad en México (Seminario: Los Factores del Cambio Demográfico en México), Universidad Nacional Autónoma de México.

Quilodrán, Julieta, 1983. Niveles de fecundidad y patrones de nupcialidad en México, El Colegio de México.

Secretaría de Salubridad y Asistencia, 1988. Encuesta Nacional sobre Fecundidad y Salud, 1987. Memoria de la reunión celebrada el 18 de febrero.

Secretaría de Programación y Presupuesto, 1979. Encuesta Mexicana de Fecundidad. Primer informe nacional, México, marzo. 